\section{Amalgam-Intoleranz oft psychisch bedingt}

Mögliche Schäden durch Amalgam werden immer noch kontrovers diskutiert. Echte Allergien sind jedoch nur bei wenigen Patienten belegt. Österreichische Mediziner haben nun untersucht, ob bei Patienten, die über Amalgam-Intoleranz klagen, Besonderheiten der Persönlichkeit vorliegen. Das Ergebnis: Typisch für ,amalgamintolerante“ Patienten ist offenbar eine geringe Lebenszufriedenheit und eine hohe allgemeine Angstbereitschaft. Außerdem gehen häufig Scheidung, Tod eines nahen Verwandten, Verlust des Arbeitsplatzes oder schwere Krankheit der Unverträglichkeit voraus.

Einbezogen in die Studie waren 25 Patienten mit Amalgam-Intoleranz-Symptomen. In Fragebögen wurden verschiedene psychologische Persönlichkeitsparameter ermittelt und mit den entsprechenden Angaben von 29 Kontrollprobanden verglichen.

Auffallend war auch, daß die Patienten mit vermeintlicher Amalgam-Intoleranz vergleichsweise oft den Behandler gewechselt hatten. Außerdem klagten die Patienten noch über verschiedene weitere Beschwerden, Kopfschmerzen, Verkrampfungen, Trockenheit und Brennen im Mund und Beschwerden beim Kauen.

Im Vergleich zur Kontrollgruppe zeigten die Amalgam-Patienten deutlich häufiger psychosomatische Störungen. Nur bei einem Patienten wurde eine echte Amalgam-Unverträglichkeit bestätigt. Zwischen der Zahl der Amalgamfüllungen in Mund, Amalgam-Unverträglichkeit und Persönlichkeitsprofil war keine direkte Korrelation feststellbar. Die psychischen Auffälligkeiten waren daher wohl nicht Folge einer chronischen Amalgam-Intoxikation.

Quelle: Kreyer G: zm 1998; 88: 2388.

\section{Neurodermitis durch hartes Wasser?}

\author{
Bei Grundschülern steigt die Ekzemneigung mit dem Härtegrad \\ des Wassers. Dies ergab eine Studie der Universität Nottingham. \\ Bei Schülern der Sekundarstufe I war dagegen keine Korrelation \\ mehr nachweisbar.
}

Erfolg und Mißerfolg hängen bei epidemiologischen Studien oft von der richtigen Fragestellung ab. Ein Beispiel hierfür ist eine Untersuchung in Nottingham, bei der die Häufigkeit von atopischen Erkrankungen unter 8000 Schülern der Grundschule (4 bis 11 Jahre) und der Sekundarstufe I (11 bis 16 Jahre) ermittelt werden sollte.

Das Ergebnis war wenig spektakulär: Asthmaerkrankungen sind im Grundschulalter bei Jungen häufiger, in der Pubertät erkranken mehr Mädchen. Außerdem wurde die hohe Prävalenz von atopischen Erkrankungen bestätigt, die bereits andere Studien gezeigt hatten. Die Ergebnisse wurden zur Kenntnis genommen. Eine größere Breitenwirkung dürfte Nick McNelly erzielen. Er nutzte die Daten der Umfrage und korrelierte sie mit den Härtegraden des Leitungswassers.

Die Auswertung ergab, daß in Regionen mit Härtegrad 4 (mehr als $231 \mathrm{mg}$ Mineralsalze/l) im letzten Jahr deutlich mehr Grundschüler an einem atopischem Ekzem erkrankt waren (17,3\% der Befragten) als in den Bezirken mit der niedrigen Wasserhärte 1 (unter $135 \mathrm{mg} / \mathrm{l}, 12 \%$ ). Bei den Schülern der Sekundarstufe I war ein Einfluß der Wasserhärte auf die Ekzemneigung nicht mehr nachweisbar.

Die Erklärung des Autors: Der Härtegrad hängt in erster Linie von der Konzentrationen an Kalzium- und Magnesiumionen ab. Sie bilden mit den Fettsäuren von Seifen unlösliche Komplexe, was bei hohen Härtegraden die Schaumbildung deutlich erschwert.

Die Kinder könnten geneigt sein, größere Mengen Seife zu verwenden, um die gewünschte Wirkung zu erzie-

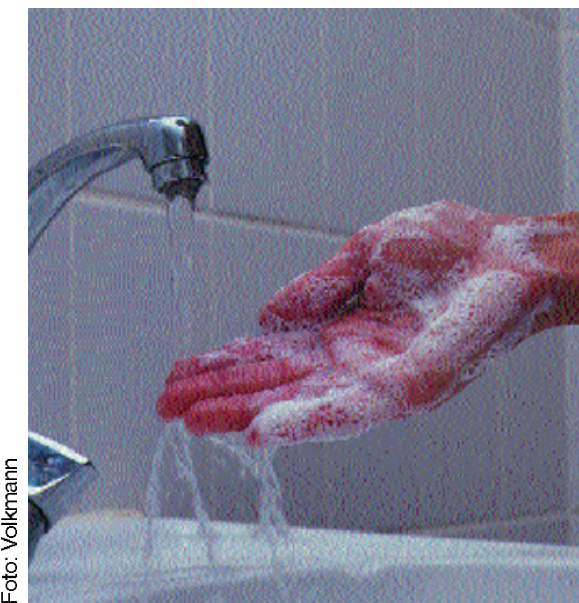

Bei hartem Wasser braucht man mehr Seife, damit es richtig schäumt. Empfind liche Kinder reagieren darauf mit Haut reizungen und in der Folge kann es zur Manifestation einer atopischen Dermatitis kommen - so die Theorie.

len. Der Mehrverbrauch an Detergenzien könnte die Haut austrocknen und die mukokutane Barriere schädigen. Dadurch erhöhe sich das Risiko, daß es bei empfindlichen Kindern zu einer Irritation der Haut kommt. Wenn die Hautbarriere erst einmal geschädigt ist, könnten Magnesium und Kalzium als Irritanzien wirken.

Anfällig für dieses Phänomen sind vor allem jüngere Kinder. Während der Pubertät scheint die Haut eine Resistenz gegen eine Störung der Hautbarriere durch die Detergenzien zu entwickeln, die Wasserhärte spielt bei diesen Kindern offenbar keine entscheidende Rolle mehr.

(rme)

Quelle: McNally $\mathrm{N} \mathrm{J}$ et al.: Atopic eczema and domestic wate hardness. Lancet 1998; 352: 527-31. 